\title{
PROGRAMA DE ACOLHIMENTO AO ESTUDANTE INGRESSANTE NO ENSINO SUPERIOR NO CONTEXTO DA PANDEMIA DA COVID-19
}

DOI: $10.37702 / 2175-957 X . C O B E N G E .2021 .3578$

CASSIA SOUZA GUERREIRO - kcisza@gmail.com

Instituto Tecnológico de Aeronáutica

ALAMEDA JOSE ALVES DE SIQUEIRA FILHO 12

12245-492 - São José dos Campos - SP

Cristiane Pessôa da Cunha Lacaz - clacaz@ita.br

Instituto Tecnológico de Aeronáutica ITA

H17B casa 102

12228-530 - São José dos Campos - SP

Daniela de Castro Silva - danicastrorh@gmail.com

Instituto Tecnológico de Aeronáutica

Rua Rosa de Toledo 123

12224-850 - São José dos Campos - SP

João Francisco Mazariolli - joaomaza@ita.br

Instituto Tecnológico de Aeronáutica

Praça Mal Eduardo Gomes 50

12228-900 - São José dos Campos - SP

Resumo: $O$ presente relato aborda a experiência de quatro propostas que constituem o Programa de Acolhimento ao estudante ingressante no ensino superior, considerando a necessidade em acolher o aluno ingressante e facilitar a sua adaptação em uma Instituição Pública Federal, que oferta cursos em seis áreas de Engenharia. As quatro propostas são: Projeto Novo Aconselhamento; Plantão de Orientação Educacional; Plantão de Acolhimento; e uma Disciplina obrigatória que já era ofertada, denominada Colóquios (FND-01) e que agora, diante das peculiaridades impostas pela pandemia, passa a ter um caráter integrador mais amplo. O Programa de Acolhimento foi implementado e referendado pela comunidade acadêmica da Instituição, que indicou viabilidade e continuidade da proposta. A experiência está revelando a importância da estratégia como uma 
forma de apoiar a adaptação e a integração ao ensino superior, diante dos desafios vividos pelos alunos ingressantes. Objetivamos que o espaço acadêmico se torne um ambiente positivamente desafiador e propício para o desenvolvimento de habilidades diversas, cada vez mais, requeridas na formação do engenheiro.

Palavras-chave: Educação Superior; integração acadêmica; adaptação ao ensino superior 


\section{(C) COBENGE \\ 28 a 30 de SETEMBRO \\ PROGRAMA DE ACOLHIMENTO AO ESTUDANTE INGRESSANTE NO ENSINO SUPERIOR NO CONTEXTO DA PANDEMIA DA COVID-19}

\section{INTRODUÇÃO}

O presente artigo discorre sobre o Programa de Acolhimento ao estudante ingressante no ensino superior, em uma Instituição Pública Federal, que oferta cursos em seis áreas de Engenharia. De acordo com Honório et al (2019), no que diz respeito ao ingressante no ensino superior, é necessário que compreenda-se que esse processo de adaptação envolve não apenas o estudante, mas também a instituição de ensino, no intuito de que esta possa atuar como facilitadora através da criação de ferramentas e processos preventivos e intervenção, se for o caso, para facilitar a adaptação dos discentes.

No ingresso para o Ensino Superior, há uma proposta para uma condução mais autônoma da aprendizagem, sendo demandando do aluno que ele seja o principal responsável por suas próprias escolhas e tomada de decisões, sendo imprescindível que esteja ciente dos processos que envolvem e podem interferir na aprendizagem e consequentemente, impactar no sucesso acadêmico (Burochovitch et al, 2017).

Complementando a ideia, conforme aponta Almeida (2019), o Ensino Superior constitui um conjunto de expectativas sociais, resultando em uma série de exigências e desafios para os ingressantes, seja em relação a carreira, seja relacionado a questões sociais e culturais e não há evidências de que, necessariamente, os novos discentes estejam prontos para atender essas demandas.

A experiência das autoras e as inúmeras pesquisas sobre o tema demonstram que, ao ingressar no ensino superior, os alunos são cobrados e necessitarão se adaptar a novas regras, novos modos de aprendizagem, além da necessária postura mais ativa em busca do conhecimento (Soares \& Del Prette, 2015; Martins \& Santos, 2018), o que são desafios para os quais nem todos os estudantes estão preparados (Araújo \& Almeida, 2015).

Em paralelo aos desafios comumentes enfrentados pelo estudante universitário, para os ingressantes neste nível de ensino a partir do início de 2020, destacamos ainda a pandemia de Covid-19. De acordo com a Organização Mundial de Saúde (2020), a pandemia teve início em 31 de dezembro de 2019, a partir da notificação de casos de pneumonia na cidade de Wuhan, na China, em decorrência de uma nova cepa de Coronavírus, que posteriormente foi confirmada como sendo um novo tipo de vírus, nomeado de SARS-CoV-2. Contudo, já no mês de janeiro de 2020, a OMS emitiu um alerta de emergência de saúde pública de nível internacional, por causa da velocidade com a qual o vírus se espalhava.

De acordo com estudos da UNESCO (2020), em todo o mundo, os sistemas educacionais foram e continuam sendo fortemente afetados pela pandemia da Covid-19. Em mais de 150 países, a pandemia produziu fechamento generalizado de instituições de ensino, em todos os níveis de escolaridade, determinando adaptações na oferta de conteúdos através de ferramentas de aprendizado não presencial.

Assim, os alunos ingressantes ficaram impossibilitados de ter proximidade física com colegas, professores e equipe administrativa que, até o mês de março de 2020 aconteciam de maneira presencial na Instituição. Ou seja, rapidamente, todas as disciplinas e iniciativas oferecidas ao corpo discente se adequaram, passando a serem oferecidas no formato on-line, preservando a integridade física de todos os envolvidos, sem prejudicar o acesso às ferramentas facilitadoras para adaptação dos discentes. 


\section{DESENVOLVIMENTO}

Considerando a necessidade em acolher o aluno ingressante e facilitar a sua adaptação ao Instituto, foram definidas quatro iniciativas como estratégias para o processo de Integração ao Ensino Superior, sendo elas: Projeto Novo Aconselhamento; Plantão de Orientação Educacional; Plantão de Acolhimento; e uma Disciplina obrigatória que já era ofertada, denominada Colóquios (FND-01) e que agora, diante das peculiaridades impostas pela pandemia, passa a ter um caráter integrador mais amplo.

\section{Projeto Novo Aconselhamento}

Desde os anos iniciais de sua criação, a Instituição em questão mantém como elemento da orientação educacional, um sistema de aconselhamento descrito em um manual de autoria de Daniel Antipoff (1964). O Aconselhamento consiste na designação de um professor-conselheiro que irá orientar o aluno durante sua trajetória acadêmica e ajudar a encontrar soluções para alguma dificuldade, além do acompanhamento em eventuais problemas de ordem pessoal.

Para Casanova (2012) a tutoria pode ser entendida como uma técnica adotada durante o processo de aprendizagem com duas finalidades distintas, designadamente como uma técnica preventiva, auxiliando na construção e desenvolvimento de diversas aprendizagens antes de as dificuldades surgirem e uma técnica remediativa, como auxiliar na superação das dificuldades detectadas.

Por meio de pesquisas e contato com outros modelos consolidados no contexto universitário, identificamos a necessidade de revitalização do sistema de aconselhamento. O novo aconselhamento é um projeto inspirado no "Programa de Tutorado" do Instituto Superior Técnico de Lisboa (IST), pioneiro em Portugal no desenvolvimento e implementação de atividades de tutoria adaptadas à realidade do ensino superior local e referência premiada de boas práticas no contexto universitário europeu. A partir da aproximação com o modelo já consolidado do Instituto Superior Técnico de Lisboa (IST), foram planejadas as ações de revitalização do aconselhamento na Instituição. Vale registrar que, diante da longa relação da Instituição com o termo "aconselhamento", optamos pela manutenção do mesmo, apesar de reconhecermos, apoiadas na literatura sobre o tema, que este deveria ser substituído por "tutoria".

Inicialmente foi realizada uma pesquisa com os estudantes regularmente matriculados na Instituição, tendo 637 respondentes. Os resultados apontaram fragilidades no modelo até então aplicado (antigo aconselhamento): número elevado de alunos sem conselheiro, sendo a maior parte dos anos iniciais; número reduzido de conselheiros somada a concentração excessiva de alunos; os dados apontaram que a iniciativa do contato, quando existia, partia quase sempre do aluno. Os dados revelaram a inefetividade e fragilidade do modelo, além da indefinição de papéis no aconselhamento e pouco apoio institucional aos Conselheiros.

A partir de então, foram iniciadas as ações de implantação. A primeira delas foi a sensibilização e lançamento de edital para inscrição dos docentes, capacitação dos professores, recepção e integração dos alunos e início das reuniões de aconselhamento a partir dos roteiros elaborados pela equipe técnica do projeto.

Ao longo dos últimos anos, são vários os estudos sobre a temática das tutorias com o objetivo de compreender os fatores que contribuem para os seus potenciais benefícios e eficácia (Silva \& Freire, 2014).

Em seu segundo ano de implantação, o novo aconselhamento já conta com 55 professores da Instituição fortemente envolvidos e abrange em torno de 300 alunos. Para a capacitação dos docentes foram convidados especialistas acadêmicos, 
médico-psiquiatra, psicólogos, juízes de direito e pedagogos. Os conteúdos ministrados abordaram os seguintes temas: saúde mental; acompanhamento pedagógico; acolhimento e integração no ensino superior; conciliação e mediação de conflitos; habilidades sociais; projeto profissional e de vida; intervenção em vulnerabilidade socioeconômica; autorregulação da aprendizagem; e, ética e disciplina consciente na vida acadêmica.

A ação tutorial exige do docente-tutor, assim como demonstrado por Cabrero et al (2016) competências que diferem da prática docente que ocorre em contexto de sala de aula, nomeadamente a escuta ativa, a empatia, o apoio individualizado e ausência de julgamento crítico superior.

\section{Plantão de Acolhimento Psicológico}

O plantão psicológico tem como proposta criar um espaço de acolhimento, onde o discente possa, de maneira autônoma, ressignificar suas experiências, seu caminho e refletir sobre si, para que assim, consigam seguir seu caminho, apesar dos obstáculos encontrados, conforme Rebouças e Dutra (2010). Assim, é importante destacar que no plantão de acolhimento o propósito é que o Psicólogo atue como facilitador do processo e não apenas como aquele que detém o saber técnico, propiciando um espaço de troca e (Oliveira, 2006),

Dentro desse contexto, o Plantão de Acolhimento Psicológico (PAP) é um espaço para que o aluno possa (re)pensar suas demandas e inquietações e assim, encontrar novas formas de pensar e agir. A proposta do PAP não é oferecer um atendimento psicoterápico clássico e sim, fornecer suporte nas demandas emergenciais que podem impactar seu desempenho acadêmico e/ou sua qualidade de vida. Caso emergências, que exigem atendimento clínico especializado, são encaminhados para os serviços de saúde específicos.

Qualquer aluno que identificar necessidade de suporte pode ser atendido. Para solicitar o atendimento no PAP, o aluno deve acessar um link amplamente disponibilizado e preencher o formulário com as seguintes informações: nome, e-mail, telefone, curso, turma, além de datas e horários desejados para o atendimento.

Após o preenchimento do formulário on-line, o aluno receberá em seu e-mail a confirmação do atendimento, com o dia, horário e plataforma onde ocorrerá o encontro. Tanto no Plantão de Acolhimento Psicológico como no Plantão de Orientação Educacional, o discente será atendido em um encontro e poderá retornar para atendimento, se for necessário, por até mais dois encontros.

Em qualquer etapa do atendimento, quando identificada a necessidade de acompanhamento por profissional especializado (psiquiatra, clínico geral, psicoterapeuta, nutricionista, etc), o discente é encaminhado e se necessário, recebe suporte para o agendamento com o profissional de saúde, seja na rede pública ou privada.

Assim sendo, destaca-se que a proposta do plantão é acolher o sofrimento do discente, sem a proposta de uma atuação clínica tradicional, mas considerando o plantão como espaço para novas possibilidades, através do oferecimento de um espaço seguro onde o aluno é acolhido e legitimamente percebido (Rebouças e Elza, 2010).

\section{Plantão de Orientação Educacional}

Os estudantes são, em sua maioria, usuários de ferramentas de comunicação digital, sendo comunicadores on-line nativos, mas nem sempre possuem habilidades e conhecimentos necessários para utilizar a internet de forma a encontrar e integrar as informações, de forma organizada e coerente, utilizando a ferramenta como facilitadora 
do conhecimento, ao invés de ser usado como fator que contribui para distração (Alexandrini e Rufini, 2017).

É importante ressaltar que a fundamentação teórica utilizada neste atendimento é baseada na autorregulação da aprendizagem, podendo ser definida, de maneira resumida, como: "um processo de autorreflexão e ação no qual o aluno estrutura, monitora e avalia o seu próprio aprendizado" (GANDA e BORUCHOVITCH, 2018 apud ZIMMERMAN \& SCHUNK, 2008).

O Plantão de Orientação Educacional (POE) é realizado de forma on-line, mediante solicitação de atendimento realizada pelo aluno. O modelo do atendimento é baseado na Teoria da Autorregulação da aprendizagem. De acordo com Burochovith et al. (2017), a aprendizagem autorregulada é definida como "a capacidade de o estudante se responsabilizar pelo planejamento e condução de sua própria aorendizagem". Deste modo, os processos auto regulatórios podem ser ensinados, para que o aluno aprenda a aprender e seja capaz de pensar de maneira crítica. Neste contexto, o aluno é atendido pelo orientador Educacional, que fornece ferramentas para que o aluno possa se organizar e se tornar o protagonista de seu processo de aprendizagem, usando o meio e as ferramentas disponíveis para estruturar suas tarefas e direcionar suas ações.

\section{Disciplina Colóquios}

Conforme apresentado no Catálogo dos Cursos de Graduação (2020), a disciplina Colóquios (FND-01) é ministrada semanalmente aos alunos ingressantes durante o primeiro semestre do $1^{\circ}$ ano da graduação, com a proposta de facilitar a integração dos alunos através da apresentação das normas e diretrizes acadêmicas, bem como da estrutura que envolve a vida universitária dentro da Instituição. Os temas abordados na disciplina são: integração à vida universitária; principais Normas da Graduação e suas implicações na vida acadêmica; o sistema de aconselhamento; disciplina consciente; projetos de pesquisa; iniciação científica e iniciação tecnológica; mudança de especialidade; outros temas propostos e construídos em sala de aula.

Dentro da programação da disciplina, além dos encontros sobre as normas da Instituição, ocorre também a apresentação das disciplinas que serão ministradas durante os cinco anos de curso, sendo que o curso fundamental corresponde aos dois primeiros anos da graduação em Engenharia no Instituto e o curso profissional, que são os três anos cursado após o curso Fundamental, na Engenharia específica escolhida pelo aluno, que pode ser: Engenharia Aeronáutica, Aeroespacial, Civil-Aeronáutica, Computação, Eletrônica ou Mecânica.

Após a conclusão da disciplina FND-01, espera-se que o aluno consiga identificar as diretrizes, normas e estruturas do curso e da Instituição e que tal conhecimento seja um facilitador na adaptação, a medida em que fornece informações básicas sobre o funcionamento do Instituto.

As aulas são ministradas pela Chefia da Divisão de Assuntos Estudantis e contam com a participação dos professores do Instituto, permitindo que discentes tenham a oportunidade de interagir com diversos integrantes da Instituição, facilitando a comunicação e interação com diversos setores acadêmicos e administrativos.

\section{CONSIDERAÇÕES FINAIS}

Atuar de maneira preventiva no sentido de oferecer ao aluno ingressante ferramentas para facilitar a integração e adaptação é imprescindível para que o processo de acolhimento seja eficaz.

Nesse sentido, o Programa de Acolhimento é uma estratégia que tem se mostrado efetiva para facilitar o processo de transição para o ensino superior, através dos 
feedbacks recebidos de alunos e professores e da aceitação do projeto do Novo Aconselhamento, Plantões de Acolhimento e Orientação Educacional.

O Aconselhamento tornou-se um importante aliado no enfrentamento às mudanças trazidas pela pandemia Covid-19, sendo os docentes estimulados a manterem o contato virtual com os alunos. O Novo Aconselhamento segue avançando em suas atividades, mantendo o pilar da capacitação contínua dos Professores-Conselheiros, o contato regular com os alunos, diálogo constante com os diversos atores institucionais e o fortalecimento da rede de apoio ao aluno.

No que diz respeito ao POE e ao PAP, os alunos têm encontrado nesses Plantões um espaço de orientação, acolhimento e direcionamento tanto para atividades acadêmicas quanto para questões sociais, emocionais e relacionais, que atuam de maneira integrada na vida do discente.

Observa-se ainda que a disciplina FND-01 tem sido um facilitador no que diz respeito ao conhecimento das normas, estrutura e particularidades da Instituição, proporcionando orientações claras e diretivas, bem como favorecendo a integração entre alunos, professores e equipe administrativa, mesmo durante o período de afastamento social, minimizando os impactos causados pelo distanciamento físico e proporcionando uma aproximação social através das aulas on-line e discussões em grupo.

\section{REFERÊNCIAS}

ALMEIDA, L.S. (2019) Ensino superior: combinando exigências e apoios. In: Almeida, L.S. Estudantes do Ensino Superior: Desafios e oportunidades. ADIPSIEDUC: Braga, 2019.

ANTIPOFF, Daniel. Pequeno Manual do Conselheiro do ITA. 1964.

ARAUJO,, A. M., \& ALMEIDA, L. S. (2015). Adaptação ao ensino superior: O papel moderador das expectativas acadêmicas. Lumen Educare, 1(1), 13-32. doi:

10.19141/2447-5432/lumen.v1.n1.p.13-32

BORUCHOVITCH, E, AZZI R, G. \& SOLIGO, A. (Orgs). Temas em Psicologia Educacional: contribuições para a formação de professores. Mercado das Letras, Campinas, SP, 2017,268 pgs

CABRERO, B. G., CEBALLOS, C. P., VIGILI, M. H. G., NIEBLA, J. C., GARDUÑO, C. M., SOTO, Y. M., RODRIGUEZ, A. S., CERVANTES, D. I., SÁNCHEZ, S. M., \& VILLANUEVA, Y. A. (2016). Las competencias del tutor universitario: Una aproximación a su definición desde la perspectiva teórica y de la experiencia de sus actores. Perfiles Educativos, 38(151), 104-121.

CASANOVA, M. P. (2012). O Papel da Tutoria no Desenvolvimento Curricular. In XIX Congresso da AFIRSE "Revisitar os Estudos Curriculares: Onde Estamos e para Onde Vamos?". EDUCA/Secção Portuguesa da AFIRSE.

GANDA, D.R.; BORUCHOVITCH, E autorregulação da aprendizagem: principais conceitos e modelos teóricos. Revista Psicologia da Educação. Programa de Estudos Pós-Graduados em Educação: Psicologia da Educação. Disponível em https://revistas. pucsp.br/psicoeduca/article/view/39147/26520. Acesso em: 06 mai. 2021. 
GUSSO, Hélder Lima et al . Ensino Superior em tempos de pandemia: diretrizes à gestão universitária. Educ. Soc., Campinas , v. 41, e238957, 2020 . Available from <http://www.scielo.br/scielo.php?script=sci_arttext\&pid=S0101-73302020000100802\&lng= en\&nrm=iso>. access on 22 Apr. 2021. Epub Sep 25, 2020. http://dx.doi.org/10.1590/es.238957.

HONÓRIO, A. C., Ottati, F., \& Cunha, F. A. (2019). Avaliação da adaptação ao ensino superior. Psicologia para América Latina, (32), 97-105.

LACAZ,C.P.C; MAZARIOLLI, J.F. A orientação educacional: Uma história de pioneirismo (Instituto Tecnológico de Aeronáutica - ITA). In: DIAS, C.E.S.B; TOTI, M.C.S; SAMPAIO, H; POLYDORO, S.A.J. (Orgs). Os serviços de apoio pedagógico aos discentes no ensino superior brasileiro. São Carlos: Pedro \& João Editores, 2020. p 359-371.

MARTINS, R. M. M., \& SANTOS, A. A. A. D. (2018). Intervention in Learning Strategies:

Study with New University Students. Paidéia (Ribeirão Preto), 28. doi: 10.1590/1982-4327e2839

REBOUCAS, Melina Séfora Souza; DUTRA, Elza. Plantão psicológico: uma prática clínica da contemporaneidade. Rev. abordagem gestalt., Goiânia , v. 16, n. 1, p. 19-28, jun. 2010 Disponível em <http://pepsic.bvsalud.org/scielo.php?script=sci_arttext\&pid=S1809-68672010000100004 \&lng=pt\&nrm=iso>. acessos em 23 abr. 2021.

SILVA, E., \& FREIRE, T. (2014). Programas de mentoria e promoção do desenvolvimento positivo de adolescentes. Revista Portuguesa de Educação, 27(1), 157-176.

SOARES, A. B., \& DEL PRETTE, Z. A. P. D. (2015). Habilidades sociais e adaptação à universidade: Convergências e divergências dos construtos. Análise Psicológica, $33(2)$, 139-151. doi:10.14417/ap.911

OLIVEIRA, R. G. (2005). Uma experiência de plantão psicológico à polícia militar do Estado de São Paulo: reflexões sobre sofrimento e demanda. Dissertação de mestrado, Universidade de São Paulo, SP.

ORGANIZAÇÃO MUNDIAL DA SAÚDE (OMS), Organização Pan-americana da saúde (OPAS). Folha informativa - COVID-19 (doença causada pelo novo coronavírus). $2020 \mathrm{abr}$ [acessado $2020 \mathrm{Abr}$ 17]. [cerca de 10 p.]. Disponível em: https://www.paho.org/bra/index.php?option=com content\&view=article\&id=6101: covid 19\&Itemid=875 [ Links ]

REBOUÇAS, MELINA SÉFORA SOUZA, \& DUTRA, ELZA. (2010). Plantão psicológico: uma prática clínica da contemporaneidade. Revista da Abordagem Gestáltica, 16(1), 19-28. Recuperado em 06 de maio de 2021, de http://pepsic.bvsalud.org/scielo.php?script=sci_arttext\&pid=S1809-68672010000100004\&l $\mathrm{ng}=\mathrm{pt} \& \mathrm{tlng}=\mathrm{pt}$. 
UNESCO [UNITED NATIONS EDUCATIONAL, SCIENTIFIC AND CULTURAL ORGANISATION] COVID-19 Educational disruption and response. Paris: Unesco, 30 July 2020. Disponível em: http://www.iiep.unesco.org/en/covid-19-educational-disruption-and-response-13363. Acesso em: 10 abr 2021.

\title{
RECEPTION PROGRAM TO INCOMING HIGHER EDUCATION STUDENTS IN THE CONTEXT OF THE COVID-19 PANDEMIC
}

\begin{abstract}
The present report addresses the experience of four proposals that constitute the Reception Program for students entering higher education, considering the need to welcome the incoming students and facilitate their adaptation in a Federal Public Institution, which offers courses in six areas of Engineering. The four proposals are: New Counseling Project; Educational Guidance Support; Reception Support; and a mandatory Discipline that was already offered, called Colloquiums (FND-01) and that now, in view of the peculiarities imposed by the pandemic, is having a broader integrating character. The Reception Program was implemented and endorsed by the academic community of the Institution, which indicated its feasibility and continuity. The experience reveals the importance of the overall strategy as a way to support the adaptation and integration to higher education, in the face of the challenges experienced by new students. We aim for the academic space to become a positively challenging and conducive environment for the development of diverse skills, increasingly required in the training of engineers.
\end{abstract}

Key words: Higher Education; academic integration; adaptation to higher education. 